\title{
Design and Development of Novel Channel estimation method for OFDM based Underwater communication
}

Asha M ( $\sim$ ashagsssietw@gmail.com )

GSSS Institute of Engineering Technology for Women

\section{T P Surekha}

Vidyavardhaka College of Engineering

\section{Research Article}

Keywords: Sparse channel tap delays, Doppler spread, Orthogonal Matching Pursuit, Rayleigh channel

Posted Date: March 23rd, 2021

DOl: https://doi.org/10.21203/rs.3.rs-173325/v1

License: (c) (i) This work is licensed under a Creative Commons Attribution 4.0 International License.

Read Full License 


\section{Abstract}

The ocean is a constantly changing environment whose properties are still great Challenges to Developing Effective Underwater Wireless Communication represent. The high absorption of electromagnetic and optical signals in the water limits this Use and development of underwater electromagnetic communication systems. A possible approach to wireless underwater communication over longer distances $(>100 \mathrm{~m})$, offers acoustic signal transmission, modulation methods such as frequency shift keying are also used (FSK) and phase-shift keying (PSK) are used.

In many areas the orthogonal frequency division multiplex method (Orthogonal Frequency Division Multiplexing, OFDM) is enforced for high data rates. It offers a multi-carrier transmission method Benefits in treating multipath propagation, nevertheless achieves a bandwidth efficiency similar to that of corresponding single-carrier processes. In recent studies, the OFDM methods are adopted for underwater communication. However the real time implementation is becoming a challenging part. The channel estimation plays vital role in underwater communication. In this research work, the novel sparse channel estimation in OFDM based underwater communication is proposed for long distances. The proposed algorithms are developed in fixed point manner which can be implemented in FPGA. The BER values are estimated for proposed algorithm and validated with the conventional algorithms. The proposed algorithms exhibits better results compared with the conventional algorithms.

\section{Introduction}

Since the past decade, the higher attention is towards underwater wireless communication. In different underwater applications, there is a growing need for facilitating high-speed wireless links with high reliability [1]. However, the applications of underwater communication are included oceanographic data collection, port and border security, seismic observations, maritime archaeology, offshore oil field exploration or monitoring, environmental monitoring, etc. Owing to the characteristics with favourable propagation in sound waves, the most commonly employed and practical technique is the underwater acoustic (UWA) transmission when the achievement of these attacks can be done through optical, radio, or sound (acoustic) waves [2].

Therefore, the research efforts have been included on this area. Different types of challenges are presented by the channel of UWA that involves multipath and fading, long propagation delays, potential high temporal and spatial variability, and limited bandwidth. No underwater acoustic channel is existed typically as different properties are exhibited by different bodies of water quantity. Severe degradation of performance can be faced in such type of channels of UWA with variation by implementing in hardware with a set of conservative and fixed parameters of transmission. UWA channels have included the four distinct characteristics such as Doppler spread, severe multipath with much longer delay spreads [3], frequency-dependent propagation loss, and low speed of sound propagation. In land-based radio channels, any one of these characteristics are not pronounced that makes the difficulty of underwater wireless communication comparatively and a design of dedicated system is required. 
The motion-induced Doppler distortion is the major issue in the application of OFDM for underwater channels that allows using to create the offset with non-uniform frequency in an acoustic signal of wideband frequency. Two approaches have focused on this problem in the prior work that includes adaptive synchronization and non-adaptive synchronization. A little overhead is required in adaptive synchronization but that depends on the coherence between adjacent blocks of OFDM [4]. Whereas in non-adaptive synchronization, null subcarriers are needed for achieving robustness to make the variations in channel rapidly [5]. By integrating it with estimation of a channel in the time domain or impulse response, the approach [6] is extended in this study. Performing the channel sparsing is the possibility for this motivation. An OFDM block is often larger than channel estimation and fewer than ' $K$ coefficients are represented that helps to characterize its transfer function on ' $K$ 'subcarriers. Based on ' $L$ ' equally-spaced data symbols [7] which used in [5], the time-domain response's $L<K$ coefficients with a certain number can be computed efficiently. To be applied in general underwater channel, a slight and important changes is suggested for this technique. However, the strongest signal may not be arrived firstly in this channel. Based on the truncation of magnitude for time-domain channel coefficients, sparsing is applied in an optimal way [8]. By removing the unnecessary noise in the estimation of full-size or overparameterized channel, performance results are improved if the channel is truly sparsed.

\section{Literature}

The configurations of Space-time coding and multi-input multi-output (MIMO) including the orthogonal frequency division multiplexing (OFDM)-based communication systems were initiated in the wireless communication perspective of terrestrial radio-frequency (RF) originally. In the literature reviews [9], it has been implemented for underwater communications successfully. Owing to the capacity of high information and robustness to large multipath spreads [10], [11], these methods are considering as the primary candidates for UWA systems with next generation. It will provide the benefits in terms of improvements in both performance of error rate and throughput rate through equalization processes and channel estimation [12]. To take into the account of benefits with diversity, the communication of cooperative or relay-assisted feature has also been developed to the systems of UWA because of the power or space restrictions and a performance limiting factor due to the path loss when it's impossible to the deploy the multi receive or transmit elements. For UWA relay channels, these studies have been demonstrated mostly on power allocation [13] and capacity with schemes of space time cooperative, distributed channel coding, and intersymbol interference (ISI) for adaptive relay-aided OFDM UWA communications and UWA channels [14], [15] based on the protocol of amplify-and-forward (AF) [16]. In [17], the investigation of channel equalization and estimation was done for systems of amplify-andforward cooperative relay based OFDM in channels of UWA. An efficient algorithm is improved with the use of a technique of space-alternating generalized expectation-maximization (SAGE) by considering an assumption of Rayleigh fading channels between relay, source, and destination notably in [18].

Based on the availability of reliable channel state information (CSI) and the inherent characteristics of the underwater channel, such type of systems' fundamental performance bounds are demonstrated. A crucial task is considered as the design of UWA system with the assumption of perfect channel estimation [19]. 
Because of the large number of unknown parameters as a result of Doppler spread and large delay, it is an extreme challenging task in the context of UWA communication.

With the utilization of a model of basis expansion, the estimation of doubly chosen channels of UWA was demonstrated by some of the earlier works. Here, for estimation of a channel [20], some subspace algorithms have been implemented such as ESPRIT and root MUSIC for reflecting the channel characteristics with variations of time [21]. To determine the arrivals of variant paths, a subspace technique can be developed by taking an inspiration from array processing. According to the subcarriers of pilot, the estimation of complex path gains can be done. For the channels only which have limited Doppler-spread, these techniques have been shown that they will provide the better results. By relying on the characteristic of time variation in the UWA channel, the number of unknown parameters is required to be estimated and improves substantially. On the channel estimation [22], the substantial demand is imposed. By utilizing pilots only, the dedicated equalization of intercarrier interference (ICI) [23] and estimation of channel is completed for the required channels of mild or more sever distortion of Doppler. Or else, use the decision feedback or additional pilots [24] to accomplish this.

According to the usage of UWA channels' sparse nature [25], the algorithms of channel estimation have been demonstrated in the literature. Since the arrivals of multipath is became resolvable, the underwater acoustic channels' impulse response is sparse most often by sufficiently providing the transmission bandwidth widely [26]. With the assessment of experimental results for both multi-carrier [27], singlecarrier [28] underwater transmissions, the effective output has been shown in the availability of large Doppler spread also by the algorithms of channel estimation. The testing of orthogonal matching pursuit (OMP) [29] and other different variants of basis pursuit (BP) have been done with the experimental and simulation studies. Even in the conditions of computational complexity [30], BP has been provided the best performance when compared to the OMP in the environment of UWA. By using the block coding of Alamouti's space-time with a scheme of transmit delivery in the form of one-receiver and two-transmit antennas [31], a technique of sparse channel estimation has been demonstrated in a recent work based on the assumption of underwater channels have included the all Doppler scale factors and Rayleigh fading are same. With the use of a method of expectation maximization (EM) [32] and merging with ESPRIT, the complex channel coefficients are estimated for each subcarrier by the proposed algorithm of OFDM-based non-data-aided in order to accommodate the estimation of delay based on the reflection of UWA channels' sparseness. When ESPRIT is dropped off from estimation, the impact on symbol error rate was too small significantly by the residual Doppler shift or an equivalent CFO as no Doppler spread is available in the received signal.

\section{Underwater Communication Implementation With Ofdm}

\subsection{Underwater communication}

Three significant factors are affected in the propagation of underwater acoustic channel such as timevarying multipath propagation, attenuation which improves with frequency of a signal, and low sound 
speed $(1500 \mathrm{~m} / \mathrm{s})$. At low range of frequencies, acoustic propagation for underwater is supported in the best way. For long range communication, utilized the low frequency band which automatically decreases the capacity of a channel as the attenuation improves with frequency. However, the bandwidth is highly restricted for long range communication. Underwater communication's performance is becoming worsened due to the parameters such as the ambient noise or the background noise and Doppler spread which is occurred owing to the random motion between the systems of transmission and reception. In low frequencies, noise domination is higher and it becomes lower by improving the frequency range.

Every physical path is acted like a time-varying low-pass filter in the underwater channel's impulse response. The Doppler shifting and spreading is introduced by the motion between the receiver and transmitter. The surface waves are initiated the disturbances, internal turbulence owing to ocean current, fluctuations in the sound velocity, and the random signal variations are contributed by the certain other minor phenomena.

With the improvement of frequency, attenuation is increased and noise is decayed which is illustrated in fig.4. It will lead to a variation of a signal-to-noise ratio (SNR) over the bandwidth of a signal. The expression of Signal-to-noise ratio can be mentioned below for a frequency band around the frequency at a distance: see formula 1 in the supplementary files.

Where $S_{I}(f)$ is the transmitted signal's power spectral density function, $N(f)$ is the noise function, and $A(I, f)$ is the attenuation function. Underwater acoustic propagation is imposed the fundamental restrictions additionally. The performance of underwater communication is affected significantly by the other system conditions. The bandwidth constraint is the most dominant one that is produced by the transducers of acoustic signals in which the usable bandwidth is restricted below the provided channel's bandwidth. In underwater channel, the significant components are included absorption loss and spreading loss that involves transmission loss. When communication distance is decreased, transmission loss is reduced and vice versa. For balancing the forecasted transmission loss, more transmission power is needed in long range communication.

Almost, the power is supplied through the battery for systems of underwater communication. So, the communication system is necessary to be efficient in terms of energy conservation. However, the energy consumption is relied on the transmitted bits' size directly. The efficient communication method is utilized that includes the supporting of high bit rate communication which is one of the best ways in conservation of power.

\subsubsection{Time varying multipath:}

The underwater acoustic channel is characterized the time varying multiple propagation paths. Own distance of travel has included at every path and propagation loss is come into the picture while reflection loss and travel. The cumulative channel impulse response is represented by the belowmentioned equation when each path delay and the attenuation path are represented by $₫ p(t)$ and $a_{p}(t)$ respectively. See formula 2 in the supplementary files. 
The complexity and random characteristics are included in the physical geometry of sea bed and sea surface. It's unpredictable that the motion of the transducers owing to the sea surface motion. Both parameters of by $₫ p(t)$ and $a_{p}(t)$ are expressed as random process. For a transmitted signal $x(t)$, the received signal $y(t)$ through the channel can be represented by the below equation.

\section{See formula 3 in the supplementary files.}

where, $\mathrm{n}(\mathrm{t})$ is the additive background noise. Due to the frequency and time dispersion, the selectivity of time-varying frequency is involved in the channel.

\subsubsection{Doppler spread:}

Owing to the sea surfaces and currents' random motions, Doppler spread is raised. The Doppler shift for arrival of each ray is contributed by the relative motions between receiver and transmitter. These rays are the primary source of generating the Doppler shifts where the rays are varied as they have different angles of arrival. The Doppler spread has also included the contribution of these variances. When compared to the overall Doppler spread, the expectation of resultant Doppler spread is larger. The system of receiver should have an ability to balance the resultant Doppler spread.

\subsection{Choice of OFDM parameters for underwater communication:}

The parameters of OFDM's selection is included a trade-off between different complex requirements for underwater communication. To design the systems of OFDM, three major parameters are considered such as bit rate, bandwidth, and tolerable delay spread. Bit rate is nothing but communication speed with free of errors and bandwidth is useful for communication of a system. The channel multipath is caused the tolerable delay spread.

Because of the causing of attenuation by absorption, available useful bandwidth is severely restricted for underwater communication. The attenuation is increased with the range of frequency. Low frequency is only utilized for long range communication. Let's say, $1 \mathrm{kHZ}$ bandwidth is only available for the communication range of $100 \mathrm{~km}$ in which the possible bit rate is reduced significantly. For short range communication, the larger bandwidth can get access to the high bit rate but the limiting factor is the transducer which supported the bandwidth. In case of the acoustic bandwidth, a wideband communication is to be considered as the underwater acoustic communication that is the major observation point. With the center frequency's order, the bandwidth is useful often. According to the narrowband communication in which the lower bandwidth is than the centre frequency in most of the existing principles of radio communication. For radio communication, the current existing techniques of signal processing are adopted and can't be adopted for communication of acoustic signals that leads to the requirements of complicated signal processing.

When over $1 \mathrm{~b} / \mathrm{s} / \mathrm{Hz}$ is to be reached over the channels, the bandwidth is restricted which implied the requirement for techniques of bandwidth-efficient modulation. The delay spread of channel is inversed to 
the coherence bandwidth of a channel. The band of frequencies' measure is indicated over the correlation of fading is highly done. If in case of a channel is frequency selective, narrow bandwidth is preferred to select for the sub-carriers. Owing to the Doppler spread, the coherence time of a channel is dictated the signal's duration. The Doppler spread and coherence bandwidth are largely impacted the duration of a symbol and sub-carrier spacing.

The coherence bandwidth of a channel is contrary to the delay spread. The correlation of fading is to the bandwidth of frequencies' measure implemented. The narrower bandwidth is preferred for the frequency selective fading for subcarriers but the wider bandwidth is needed for larger Doppler spreading for subcarriers. Based on both parameters like Doppler spread and coherent bandwidth, the duration of a symbol and the spacing of sub-carrier are demonstrated.

The larger guard time should be selected when compared to the delay spread of channel. For minimization of the SNR loss, the delay spread should be much shorter than the duration of a symbol. The subcarriers' spacing is very low when the larger value for symbol duration that improves the sensitivity to errors of frequency offset phase noise along with the increment of peak-to-average ratio (PAPR). The complexity would be increased with the development of signal processing. For the available bandwidth, the subcarrier spacing is chosen practically that leads to the sub-carriers' number is a tradeoff between the coherence time of channel and coherence bandwidth of channel. Based on the required bit rate, the sub-carriers' number is determined. Here, the bit rate is categorized by the bit rate of sub-carrier.

In order to increase the performance of OFDM systems, channel estimation is required. The optimum selection of parameters of OFDM is resulted with the accurate channel estimation that leads to the efficient communication system.

\section{Proposed Model}

In UWA channels with doubly-selective Rician, a technique of new pilot assisted channel estimation is proposed in this paper that incorporates the scheme of OFDM transmission. The contributions of this work is described in the below section as follows.

To increase the channel estimation algorithm's performance, the UWA channel impulse response (CIR)'s sparse structure is exploited firstly owing to the estimation of reduced number of taps. Based on a conventional OMP algorithm, the Doppler shifts and overall sparse channel tap delays are computed by the resulting algorithm primarily. Based on the combination of maximum a posteriori probability (MAP) estimation technique and the OMP algorithm, an algorithm of computationally efficient and lowcomplexity novel channel estimation is improved by using the information of Doppler spread and path delays. This technique is used for determination of complex channel path gains which include unknown variances and mean with Rician distribution of prior densities. With the use of a technique of maximum likelihood (ML), those channel path gains are estimated separately in an efficient way computationally. By averaging the parameters over the complex channel paths with Rician distribution, those parameters of likelihood function is computed. When compared to obtained results from the algorithm of OMP, the 
proposed technique is shown in figure 1 shows the performance of estimation is improved substantially based on the computer simulations.

In the proposed model, we employ the sparse channel estimation instead of the traditional channel estimation methods. Correspondingly, we have to reconsider the pilot design since the sparse channel estimation using compressed sensing (CS) techniques is essentially different with traditional methods. We perform the pilot design based on the results of spectrum sensing.

In this approach, ideal spectrum sensing without false alarm or missing detection has been assumed. After spectrum sensing, the OFDM subcarriers occupied by primary users are first deactivated. From the remaining active subcarriers, we select some to transmit pilot symbols and the others to transmit data symbols for secondary users. The resulting sequence is transformed into the time-domain signal via inverse fast Fourier transform (IFFT). To avoid inter-symbol interference, a cyclic prefix (CP) is added whose length is usually larger than the maximum channel delay spread $\mathrm{L}$.

The Algorithm for FPGA based OFDM in Underwater communication is given as:

1. Specify the Initialization parameters such as preamble length, channel length, FFT length, cyclic prefix, no of bits for transmission and frames etc.

Transmitter:

2. Variable Signal to Noise ratio is chosen as $0 \mathrm{~dB}, 10 \mathrm{~dB}, 20 \mathrm{~dB}, 30 \mathrm{~dB}, 40 \mathrm{~dB}$.

3. Generation of random data for transmission and sensing matrix

4. Apply the QPSK mapping and pass through Rayleigh channel.

5. Parameters of OFDM in underwater environment is considered (Carrier, Bandwidth and symbol duration etc)

Receiver:

1. Apply channel estimation using OMP algorithm

2. Perform data detection (Discarding preamble and cyclic)

3. De-mapping QPSK bits and calculate the BER

4. Iterate process until the no of frames completed for each SNR value.

\section{Results And Discussion}

The proposed algorithm initially estimates the overall sparse channel tap delays and Doppler shifts using a compressed sensing approach, in the form of the orthogonal matching pursuit (OMP) algorithm. Then, a computationally efficient and novel channel estimation algorithm is developed by combining the OMP 
technique for estimating the sparse complex channel path gains. The parameters considered for the OFDM in underwater environment is reported in table 1.

Table 1: Parameters considered for the OFDM in Underwater environment

\begin{tabular}{|lll|}
\hline S.No & Parameter & Value \\
\hline 1 & SNR $(\mathrm{dB})$ & $0,10,20,30,40$ \\
\hline 2 & Carrier frequency & $24 \mathrm{Mhz}$ \\
\hline 3 & Bandwidth & $8 \mathrm{Mhz}$ \\
\hline 4 & No of bits per symbol & 52 \\
\hline
\end{tabular}

The main motivation of this research work is to develop FPGA based sparse channel estimation for OFDM underwater communication. An efficient framework has been developed for FPGA based real time environment. Initially the proposed algorithms has been tested on Matlab-Simulink testbed platform. After successful implementation in Matlab-Simulink environment, these algorithms are ported on FPGA target device. The proposed algorithms are written Matlab source code and the corresponding Simulink blocks as shown in figure 3 .

In Figure 2, the initialization parameters such as, preamble length, channel length, FFT length and number of frames are declared and passed into further blocks. In the txrx block sensing matrix and transmission and reception modules are developed. The transmitted signal passed through Rayleigh channel with AWGN noise. The QPSK modulation scheme has been used for efficient data transmission. In the receiver module, the OMP algorithm is used as channel estimation technique. The $24 \mathrm{Khz}$ frequency has been used as a carrier for the transmission and it can capable to establish communication up to $50 \mathrm{~km}$ distance. The bit error rate (BER) is measured at each frame and it is considered as numerical metrics to evaluate the performance of proposed algorithm. The Simulated BER results are shown in figure 3.

In order to validate the proposed algorithms, the conventional OFDM algorithms are considered as a benchmark. From figure 3, the numerical BER values indicates that proposed algorithms which is (OMPsparse) exhibits better performance compared with the existing algorithms. In order to develop a FPGA based algorithms, the entire algorithms should converted from floating point to fixed point which is major bottle neck issue. To overcome floating point issue, the proposed algorithms are developed in fixed point manner which can be more compatible for FPGA implementation. The corresponding BER values are reported in the Table 2.

Table 2: BER metrics of Fixed point algorithms for FPGA implementation 


\begin{tabular}{|lll|}
\hline S. No & SNR $(\mathrm{dB})$ & BER \\
\hline 1 & 0 & 0.4003 \\
\hline 2 & 10 & 0.1331 \\
\hline 3 & 20 & 0.0172 \\
\hline 4 & 30 & 0.0017 \\
\hline 5 & 40 & $1.8457 \mathrm{e}-04$ \\
\hline
\end{tabular}

The numerical values from table 1 shows that fixed point FPGA based algorithms exhibits same performance as compared with floating point algorithms.

The Figure 6 shows that, bar graph representation of BER comparison values of proposed and conventional OFDM for under water communication algorithms. The comparison results indicates that, proposed algorithm provides better results compared with LMS and MP based OFDM algorithms.

\section{Conclusion}

In this research work the FPGA compatible novel channel estimation algorithm for OFDM based underwater communication is presented. In this algorithm, unknown parameters of underwater communication channel such as channel gains and delays are estimated by using OMP algorithm. The performance of the proposed algorithm is validated with the conventional algorithms. The computer simulations are carried out with different SNR values. Optimal parameters are used for the long range distance in underwater communication. The proposed algorithms are implemented in Matlab-Simulink environment.

\section{Declarations}

Please include Declaration statements for your paper. All manuscripts must contain the following sections under the heading 'Declarations'. It can be placed before the reference section.

*Funding (information that explains whether and by whom the research was supported)

'Not applicable'

*Conflicts of interest/Competing interests (include appropriate disclosures)

'Not applicable'

*Availability of data and material (data transparency) 
'Not applicable'

*Code availability (software application or custom code)

'Not applicable'

\section{References}

1. Kaushal, Hemani, and Georges Kaddoum. "Underwater optical wireless communication." IEEE access4 (2016): 1518-1547.

2. Guan, Quansheng, Fei Ji, Yun Liu, Hua Yu, and Weiqi Chen. "Distance-vector-based opportunistic routing for underwater acoustic sensor networks." IEEE Internet of Things Journa/6, no. 2 (2019): 3831-3839.

3. Qu, Fengzhong, Zhenduo Wang, Liuqing Yang, and Zhihui Wu. "A journey toward modeling and resolving doppler in underwater acoustic communications." IEEE Communications Magazine54, no. 2 (2016): 49-55.

4. Ma, Lu, Shengli Zhou, Gang Qiao, Songzuo Liu, and Feng Zhou. "Superposition coding for downlink underwater acoustic OFDM." IEEE Journal of Oceanic Engineering42, no. 1 (2016): 175-187.

5. Abdelkareem, Ammar E., Bayan S. Sharif, and Charalampos C. Tsimenidis. "Adaptive time varying Doppler shift compensation algorithm for OFDM-based underwater acoustic communication systems." Ad Hoc Networks45 (2016): 104-119.

6. Qiao, Gang, Zeeshan Babar, Feng Zhou, Lu Ma, and Xue Li. "Low-complexity progressive MIMOOFDM receiver for underwater acoustic communication." Symmetry11, no. 3 (2019): 362.

7. Uwaechia, Anthony N., and Nor M. Mahyuddin. "A review on sparse channel estimation in ofdm system using compressed sensing." IETE Technical Review34, no. 5 (2017): 514-531.

8. Li, Guyue, Aiqun Hu, Chen Sun, and Junqing Zhang. "Constructing reciprocal channel coefficients for secret key generation in FDD systems." IEEE Communications Letters22, no. 12 (2018): 2487-2490.

9. Zeng, Zhaoquan, Shu Fu, Huihui Zhang, Yuhan Dong, and Julian Cheng. "A survey of underwater optical wireless communications." IEEE communications surveys \& tutorials19, no. 1 (2016): 204238.

10. Chen, Yang, Ling Zou, Anbang Zhao, and Jingwei Yin. "Null subcarriers based Doppler scale estimation for multicarrier communication over underwater acoustic non-uniform Doppler shift channels." In 2016 IEEE/OES China Ocean Acoustics (COA), pp. 1-6. IEEE, 2016.

11. Hao, Jinxing, Jintao Wang, and Changyong Pan. "Low complexity ICI mitigation for MIMO-OFDM in time-varying channels." IEEE Transactions on Broadcasting62, no. 3 (2016): 727-735.

12. Rawat, Manishika, Brejesh Lall, and Seshan Srirangarajan. "Angle of Arrival Distribution in an Underwater Acoustic Communication Channel With Incoherent Scattering." IEEE Access8 (2020): 133204-133211. 
13. Khan, Md Rizwan, Bikramaditya Das, and Bibhuti Bhusan Pati. "Channel Estimation Strategies for Underwater Acoustic (UWA) Communication: An Overview." Journal of the Franklin Institute(2020).

14. Tran-Dang, Hoa, and Dong-Seong Kim. "Channel-aware cooperative routing in underwater acoustic sensor networks." Journal of Communications and Networks21, no. 1 (2019): 33-44.

15. Panayirci, Erdal, Mhd Tahssin Altabbaa, Murat Uysal, and H. Vincent Poor. "Sparse channel estimation for OFDM-based underwater acoustic systems in Rician fading with a new OMP-MAP algorithm." IEEE Transactions on Signal Processing67, no. 6 (2019): 1550-1565.

16. Zhang, Xiaokai, Bangning Zhang, Kang An, Zhuyun Chen, Silin Xie, Heng Wang, Long Wang, and Daoxing Guo. "Outage performance of NOMA-based cognitive hybrid satellite-terrestrial overlay networks by amplify-and-forward protocols." IEEE Access7 (2019): 85372-85381.

17. Altabbaa, Mhd Tahssin, and Erdal Panayirci. "Channel estimation and equalization algorithm for OFDM-based underwater acoustic communications systems." ICWMC 2017(2017): 121.

18. Li, Jianzhi, Bo Ai, Ruisi He, Qi Wang, Bei Zhang, Mi Yang, Ke Guan, and Zhangdui Zhong. "Directional analysis of indoor massive MIMO channels at $6 \mathrm{GHz}$ using SAGE." In 2017 IEEE 85th Vehicular Technology Conference (VTC Spring), pp. 1-5. IEEE, 2017.

19. Qiao, Gang, Lei Liu, and Lu Ma. "Analysis of outdated channel state information in underwater acoustic downlink OFDMA system." In 2018 OCEANS-MTS/IEEE Kobe Techno-Oceans (OTO), pp. 1-5. IEEE, 2018.

20. Khan, Md Rizwan, Bikramaditya Das, and Bibhuti Bhusan Pati. "Channel Estimation Strategies for Underwater Acoustic (UWA) Communication: An Overview." Journal of the Franklin Institute(2020).

21. Feng, Rui, Yu Liu, Jie Huang, Jian Sun, and Cheng-Xiang Wang. "Comparison of music, unitary ESPRIT, and SAGE algorithms for estimating 3D angles in wireless channels." In 2017 IEEE/CIC International Conference on Communications in China (ICCC), pp. 1-6. IEEE, 2017.

22. Li, Yongzhi, Cheng Tao, Gonzalo Seco-Granados, Amine Mezghani, A. Lee Swindlehurst, and Liu Liu. "Channel estimation and performance analysis of one-bit massive MIMO systems." IEEE Transactions on Signal Processing65, no. 15 (2017): 4075-4089.

23. Ma, Xuefei, Tingting Wang, Yun Lin, and Shanshan Jin. "Parallel iterative inter-carrier interference cancellation in underwater acoustic orthogonal frequency division multiplexing." Wireless Personal Communications102, no. 2 (2018): 1603-1616.

24. Karthick, R., and M. Sundararajan. "A Reconfigurable Method for TimeCorrelatedMimo Channels with a Decision Feedback Receiver." International Journal of Applied Engineering Research12, no. 15 (2017): 5234-5241.

25. Junejo, Naveed Ur Rehman, Hamada Esmaiel, Mingzhang Zhou, Haixin Sun, Jie Qi, and Junfeng Wang. "Sparse channel estimation of underwater TDS-OFDM system using look-ahead backtracking orthogonal matching pursuit." IEEE Access6 (2018): 74389-74399.

26. Altabbaa, Mhd Tahssin, Arif Selcuk Ogrenci, Erdal Panayirci, and H. Vincent Poor. "Sparse channel estimation for space-time block coded OFDM-based underwater acoustic channels." In 2018 IEEE Global Communications Conference (GLOBECOM), pp. 1-6. IEEE, 2018. 
27. Vassilieva, Olga, Inwoong Kim, Tomofumi Oyama, Shoichiro Oda, Hisao Nakashima, Takeshi Hoshida, and Tadashi Ikeuchi. "Reach extension with 32-and 64 GBaud single carrier vs. multi-carrier signals." In 2017 Optical Fiber Communications Conference and Exhibition (OFC), pp. 1-3. IEEE, 2017.

28. Tu, Xingbin, Aijun Song, and Xiaomei Xu. "Prefix-free frequency domain equalization for underwater acoustic single carrier transmissions." IEEE Access6 (2017): 2578-2588.

29. Zhang, Xin, Zhiwen Liu, Qiang Miao, and Lei Wang. "Bearing fault diagnosis using a whale optimization algorithm-optimized orthogonal matching pursuit with a combined time-frequency atom dictionary." Mechanical Systems and Signal Processing1 07 (2018): 29-42.

30. Ding, Liming, Rongfeng Li, Yongliang Wang, Lingyan Dai, and Fengbo Chen. "Discrimination and identification between mainlobe repeater jamming and target echo by basis pursuit." IET radar, sonar \& navigation11, no. 1 (2016): 11-20.

31. Toka, Mesut, and Oğuz Kucur. "Non-orthogonal multiple access with alamouti space-time block coding." IEEE Communications Letters22, no. 9 (2018): 1954-1957.

32. Tzoreff, Elad, and Anthony J. Weiss. "Expectation-maximization algorithm for direct position determination." Signal processing133 (2017): 32-39.

\section{Figures}




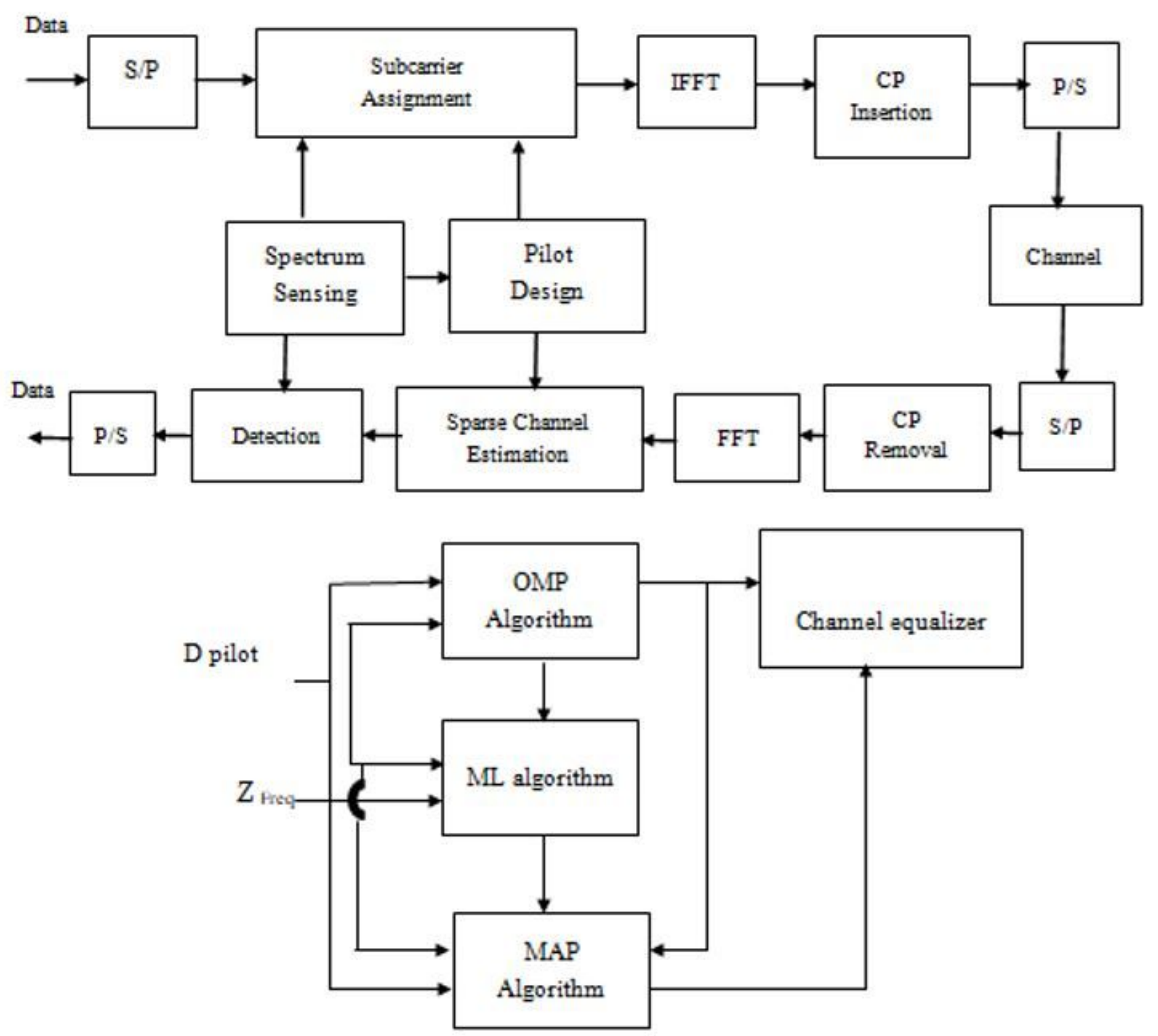

Figure 1

Block diagram of proposed model 


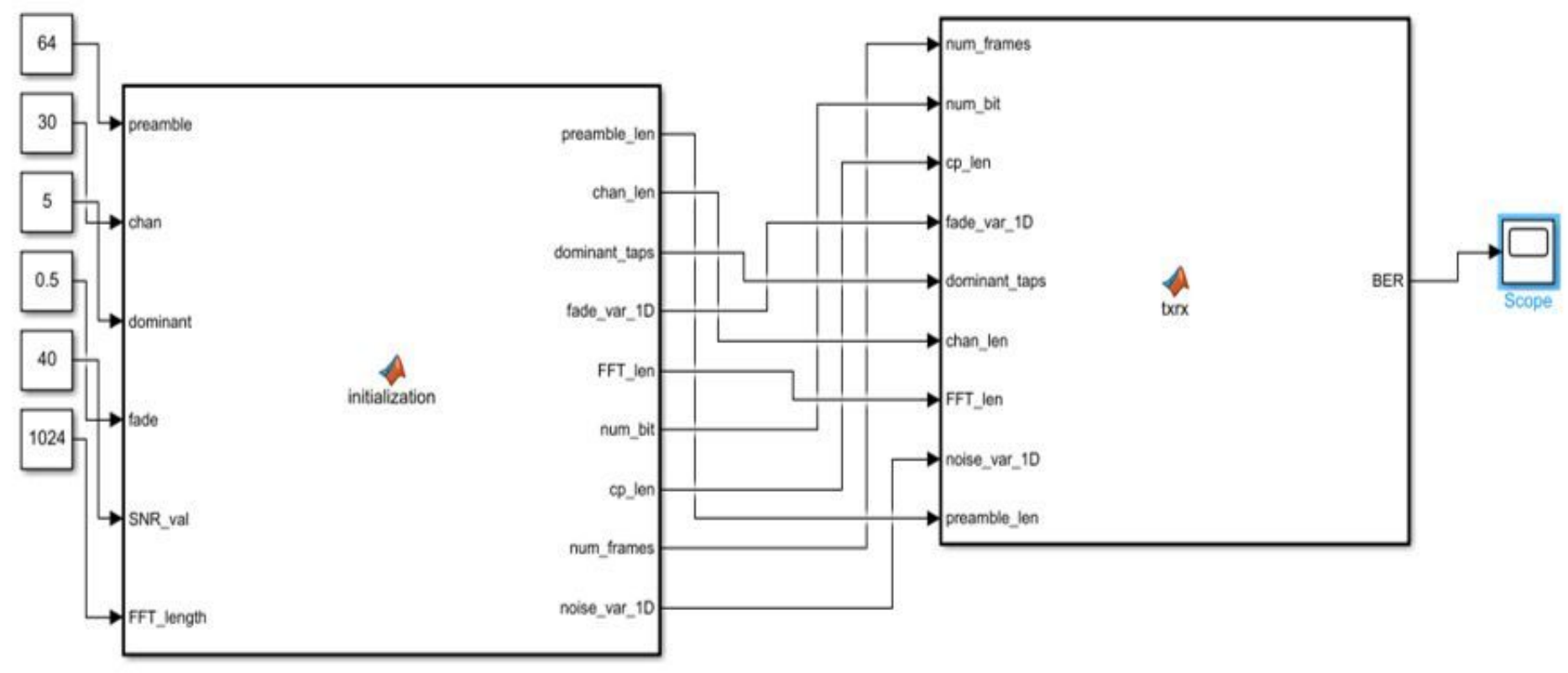

Figure 2

Proposed algorithms design blocks in Matlab-Simulink environment

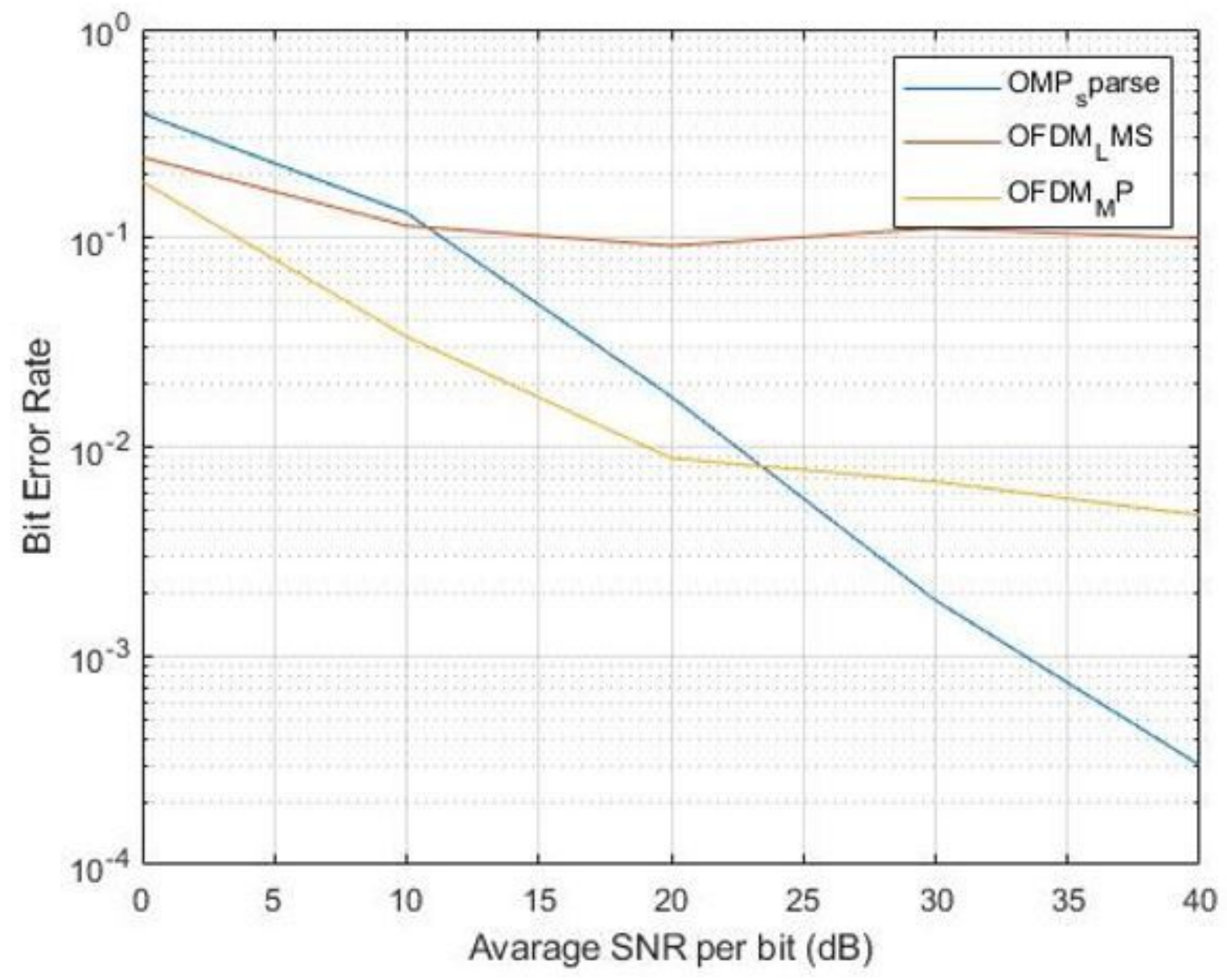

Figure 3 
Comparison results of BER vs SNR OFDM for under water communication

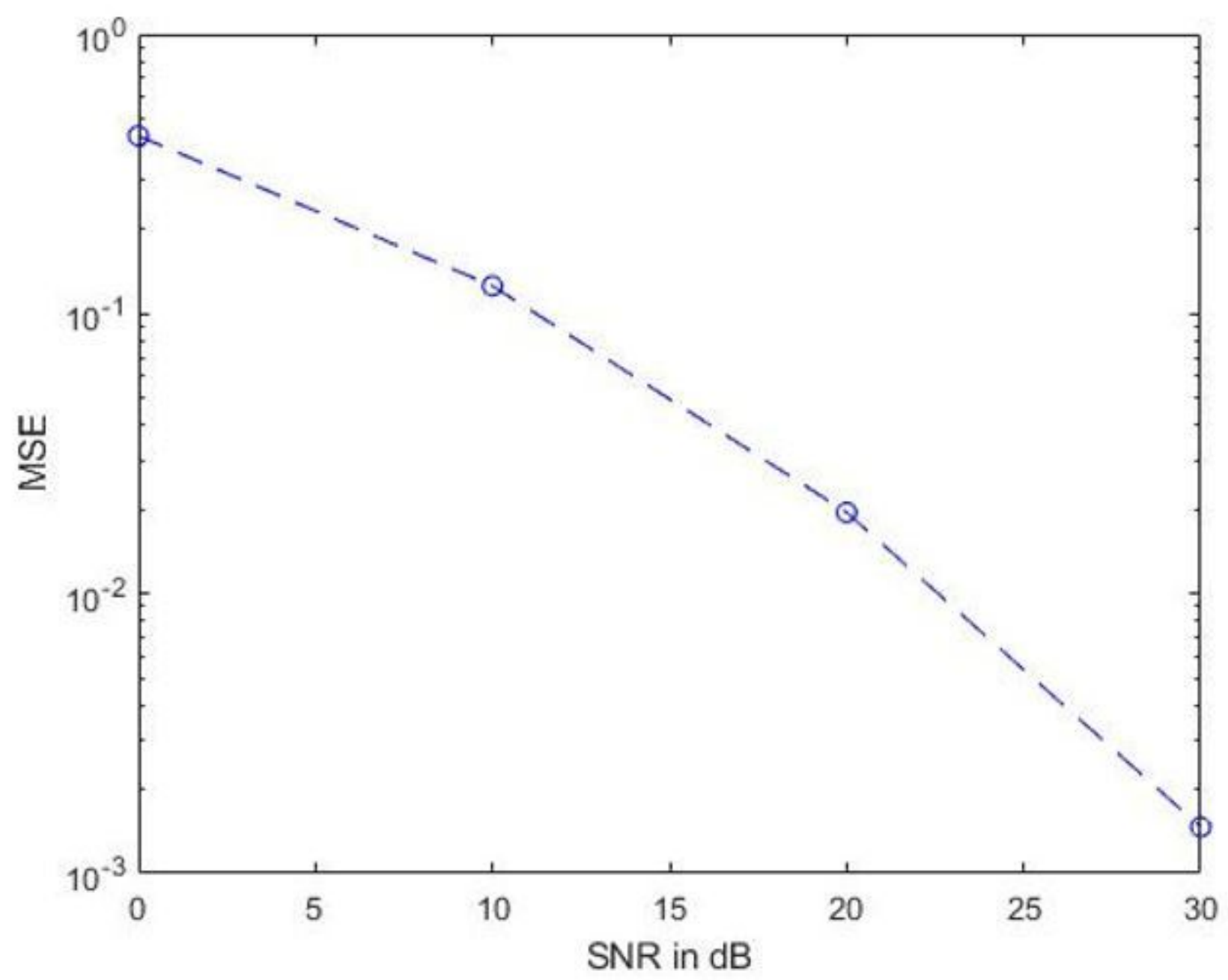

Figure 4

MSE vs SNR plot 


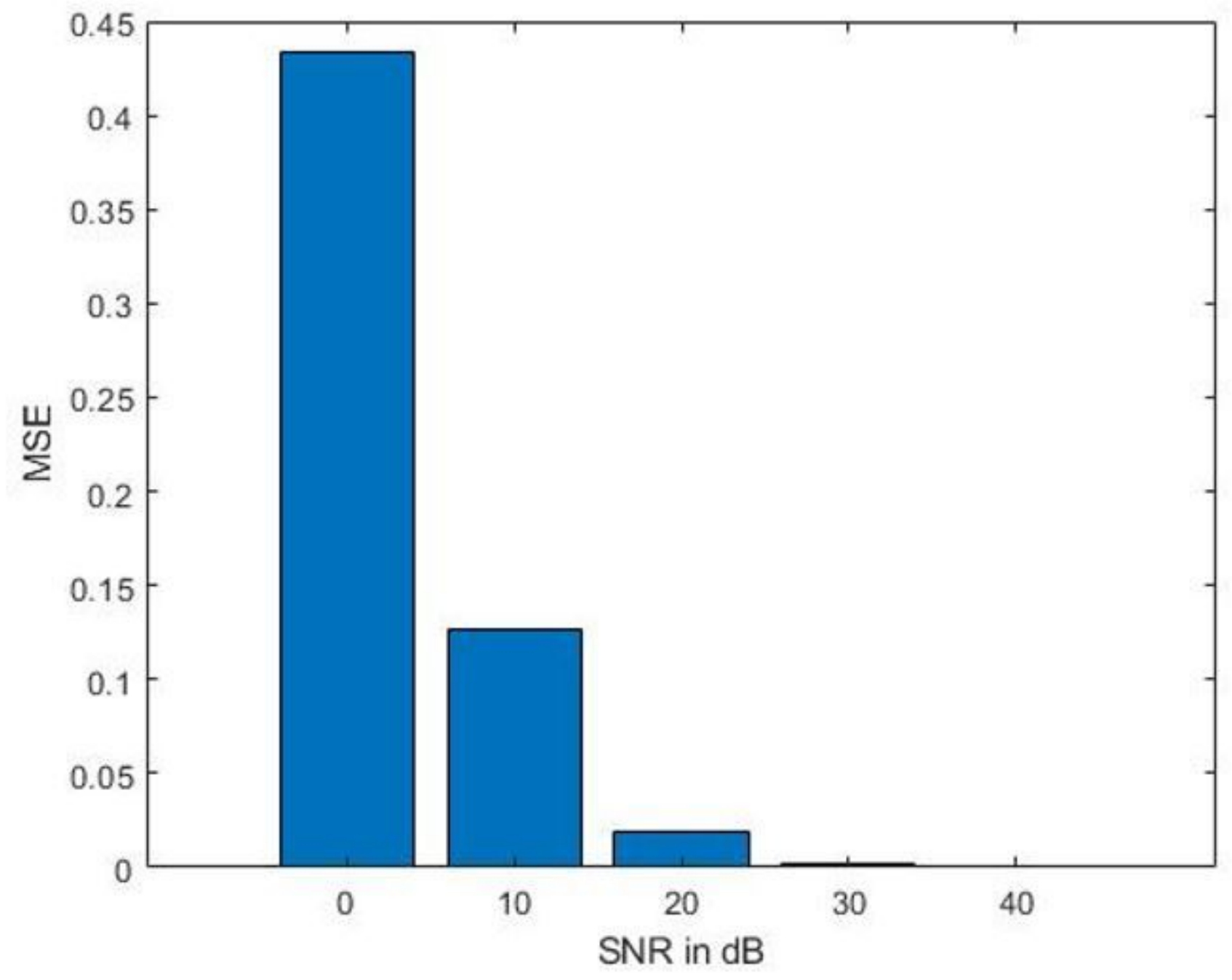

Figure 5

MSE vs SNR bargraph representation 


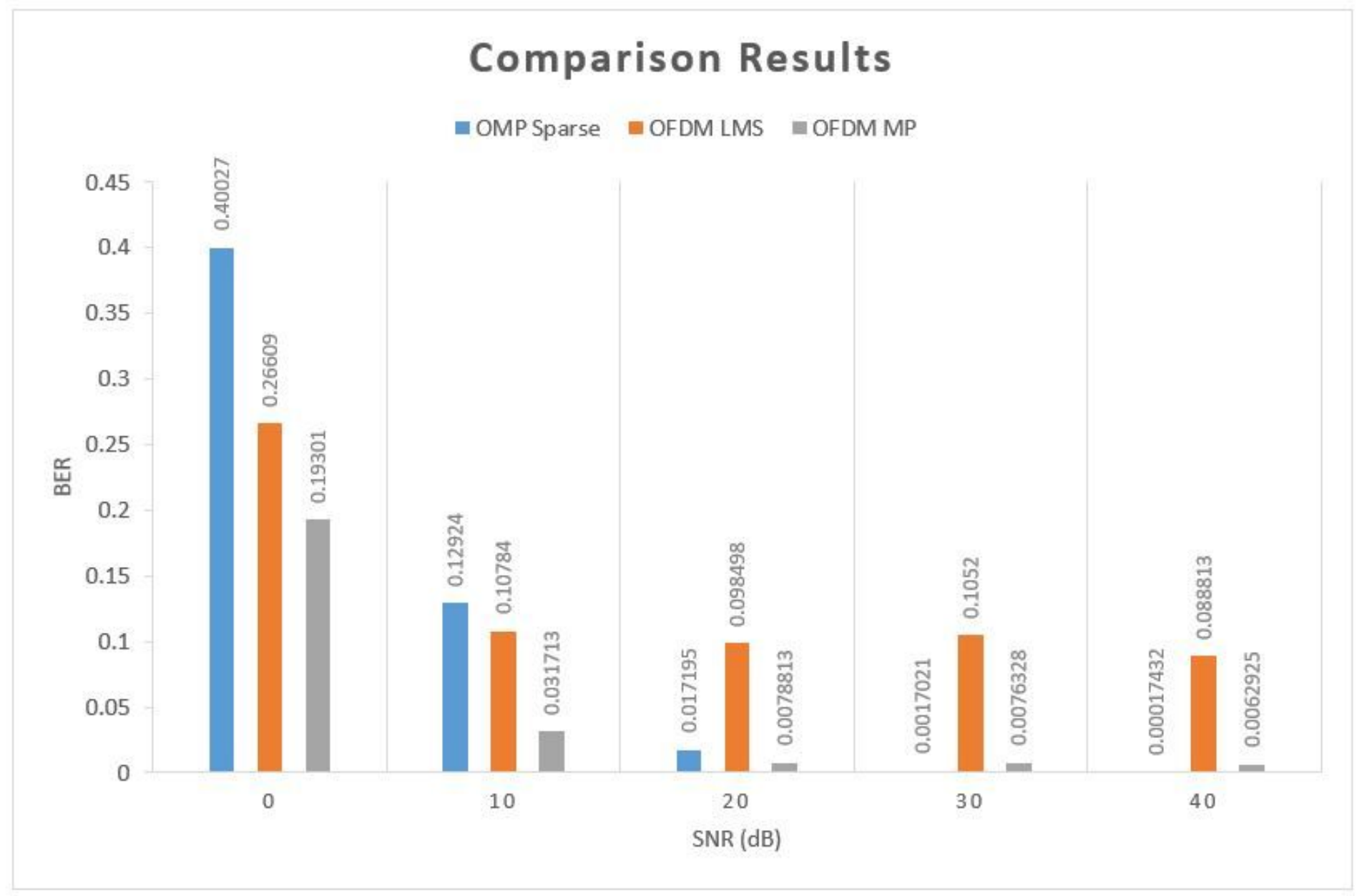

Figure 6

BER comparison bar graph chart

\section{Supplementary Files}

This is a list of supplementary files associated with this preprint. Click to download.

- formulas.docx 